\title{
Cost-Effectiveness Analysis of Ceftazidime- Avibactam Versus Colistin-Meropenem in the Treatment of Infections Due to Carbapenem-Resistant Enterobacteriaceae in Colombia
}

\section{FA Varón-Vega}

Universidad de Navarra

N Castaño

Pfizer

E Lemos

Pfizer Colombia

Juan Manuel Reyes ( $\nabla$ juanmanuel.reyes@pfizer.com )

Pfizer Colombia https://orcid.org/0000-0003-0806-7173

\section{Research}

Keywords: Cost-effectiveness, Ceftazidime-Avibactam, Colistin-meropenem, Carbapenem-Resistant Enterobacteriaceae, Colombia

Posted Date: July 7th, 2020

DOI: https://doi.org/10.21203/rs.3.rs-40162/v1

License: (1) This work is licensed under a Creative Commons Attribution 4.0 International License. Read Full License 


\section{Abstract}

Background: Ceftazidime-Avibactam (CAZ-AVI) may offer a significant advance over previously antimicrobials against carbapenem-resistant Enterobacteriaceae (CRE). We evaluate the cost-effectiveness of CAZ-AVI compared to colistin-meropenem (COL+MEM) in the treatment of CRE infections in Colombia

Methods: A decision tree model was developed from healthcare system perspective assuming a 30-day time horizon. Inputs were derived from a published observational study. The clinical course was simulated based on treatment response between 48-72 hours, and the duration of the treatment was 7-14 days. The clinical failure was assumed as the addition of an antibiotic. The model considered that combination therapy of $\mathrm{COL+MEM}$ was not superior to monotherapy to reflect real clinical behavior. Cost inputs were extracted from a published Colombian manual tariffs and official databases, expressed in 2019 dollars (USD). Utility values were from published literature. The sensitivity analyses were performed.

Results: In the base case analysis, CAZ-AVI was associated with reduced mortality, length of hospital stay and fewer add-on antibiotics, resulting in an increase of 1.76 QALYs per patient versus COL+MEM. and incremental costs associated in CAZ-AVI were $\$ 2,521$ higher per patient compared to COL+MEM (\$755 versus $\$ 3,276)$. The incremental costs were partially increased due to the lower mortality rate observed with CAZ-AVI. The incremental cost-effectiveness ratio was estimated to be $\$ 3,317$ per QALY. In the probabilistic sensitivity analysis, with a willingness to pay above $\$ 2,438$, CAZ-AVI has a higher probability of being cost-effective.

Discussion: CAZ-AVI demonstrates cost-effectiveness as a treatment for CRE infections by reducing the number of deaths and increasing QALYs.

\section{Background}

Carbapenems-resistant Enterobacteriaceae (CRE) are a serious threat to public health, being associated with high mortality rates and poor clinical outcomes (1). This may be due to a delay in treatment time, limitations in available treatment options, and the fact that patients with CRE infections tend to be critically ill (2).

The growth in resistance rates of Gram-negative bacteria has limited the benefit of the few effective and welltolerated treatment options available $(3,4)$. Ceftazidime/avibactam (CAZ-AVI) is a novel, $\beta$-lactam/ $\beta$ lactamase inhibitor fixed-dose combination drug containing ceftazidime (an established, extended-spectrum and avibactam (a unique, non- $\beta$-lactam, $\beta$-lactamase inhibitor)(5). It was launched worldwide and has demonstrated efficacy and safety through a robust Phase III clinical trial program in patients compared with carbapenems in Gram-negative infections such as complicated urinary infections $(6,7)$, complicated intraabdominal infection (8) and hospital associated pneumonia including ventilator-associated pneumonia (9). For CRE infections, CAZ-AVI has demonstrated important results through real world studies in terms of clinical and microbiological results (10), and also in reduction of mortality, failure renal, and length of stay compared to colistin (11).

The current available treatment options to tackle CRE infections in Colombia are colistin, polymyxin, tigecycline and fosfomycin, in combination with carbapenems. However, the polymyxins are associated with 
high rates of nephrotoxicity, low efficacy, and uncertainty on dosing and resistance (12-14). CAZ-AVI has recently become available as a safe alternative for Gram-negative infections including CRE $(15,16)$.

Considering that CAZ-AVI is a new agent in the country, it reflects the widespread interest in the evidence of the economic value of this treatment along with the clinical evidence. Therefore, the aim of the study to assess the cost-effectiveness of CAZ-AVI compared to colistin in combination with meropenem (COL + MEM) in the treatment of CRE infections.

\section{Materials And Methods}

Clinical management of CRE infections is complex, especially when the evidence in this population is limited. To unify the evidence of the economic value of treatments together with clinical efficacy, a simplified decision tree was developed and validated by clinicians with extensive clinical experience in the management of these infections and critically ill patients, in this way, ensure that the economic model developed reflects the clinical practice of the country. The structure, temporary horizon and assumptions used are detailed below.

Model Structure

The decision tree was designed in Microsoft Office Excel version 1908. The model was based on data derived mainly from Van Duin et al. (11). This included the 30-day hospital follow-up with hospital discharge, mortality rate, duration of initial therapy, percentage of patients who develop renal failure, and the likelihood of receiving additional antibiotics.

Figure 1. Model structure

In the model, patients begin treatment with CAZ-AVI or COL + MEM. Colistin alone was excluded as a monotherapy comparator since, in the opinion of experts, it does not reflect clinical practice where combination therapies are usually the most appropriate treatment strategies (2). Moreover, the results of the clinical study by Paul et al., showed that the combination therapy, COL + MEM, generates similar clinical outcomes compared to colistin monotherapy (17), unfortunately, the low number of patients impaired the statistical power subgroups (18), different to A baumannii infections, and there is not another study to provide conclusive evidence.

In this model, therapeutic failure is supposed to occur between 48 and 72 hours, which represents an extension of treatment (19). This failure has been defined as the addition of other antibiotics (tigecycline or fosfomycin). Patients who respond and do not require a second antibiotic will be discharged home according to the probability of occurrence of it reported by Van Duin et al. (11), and the treatment length will be 10 days as was observed in the same study. Among those who do not respond, a second antibiotic is added after 2-3 days; after this, a new 10-day course of treatment begins. Polymyxin and tigecycline would be as add-on treatment for CAZ-AVI and COL + MEM, in the proportions reported by Van Duin et al. (11). Renal failure probability used was the reported in the observational study after the 30-days of follow up.

\section{Effectiveness}


Effectiveness was expressed as quality-adjusted life years (QALY), where cohort survival is calculated after 30 days of follow-up. The life years saved were calculated by multiplying the percentage of patients alive at the end of the follow-up by the life expectancy of this type of patient. The life expectancy reported in the National Administrative Department of Statistics (DANE) (20) was adjusted to relative risk of death for survivors of 0.51 , according to a study by Garcia-Hernandez and Mayrhofer (21).

The model included two health state utilities, for patients who a clinical response 0.92 , according to a study by Song et al.(22), and for patients who had therapeutic failure 0.61 , according to the publication by Delate et al.(23).

Costs

All costs are reported in dollars (USD) 2019. The exchange rate used was COP\$3,282 per dollar (24). The costs of medications were obtained from the January to March 2019 reports of the Drug Price Information System (i.e. SISMED). For dose determination, he average weight of $65 \mathrm{~kg}$ was assumed. The daily dosage was derived for colistin from the study by Van Duin study (11); meropenem, from the study by Paul et al. (17); and the other antibiotics, including CAZ-AVI, from the study by Morrill et al. (2).

Table 1. Total treatment costs

Table 1

Total treatment costs

\begin{tabular}{|llll|}
\hline & & Daily dose & Mean cost per unit \\
\hline Treatments & & \\
\hline Meropenem, g & 6 & $\$ 6.53$ \\
\hline Colistin, million unit (MIU) & Loading dose & $3^{*}$ & $\$ 11.83$ \\
\cline { 2 - 3 } & Maintenance dose & $4.5^{*}$ & \\
\hline CAZ-AVl, g & & 7.5 & $\$ 40.23$ \\
\hline Additional treatments & & \\
\hline Tigecycline, mg & 100 & $\$ 21,61$ \\
\hline Fosfomycin, g & 12 & $\$ 2,29$ \\
\hline *Average weight $60 \mathrm{~kg}$ & & & \\
\hline
\end{tabular}

The fees in the Mandatory Traffic Accident Service (i.e. SOAT) (25) and Tariff Manual ISS 2001 (26) manuals were used for hospital costs, and include the monitoring costs and stays in ward and in the Intensive Care Unit (ICU).

The cost associated with the adverse event of treatment-related renal failure was extracted from the published literature (27), and from the Electronic System for Public Procurement (i.e. SECOP)(28). To update prices, the Consumer Price Index (CPI) reported by the Central Bank was used (29). 
Table 2. Costs inputs

Table 2

Costs inputs

\begin{tabular}{|llc|}
\hline & Mean & SD \\
\hline Hospitalization & & \\
\hline Ward & $\$ 35$ & $\$ 32$ \\
\hline ICU & $\$ 313$ & $\$ 207$ \\
\hline Adverse event & & \\
\hline Renal failure & $\$ 426$ & $\$ 145$ \\
\hline Dialysis & $\$ 1,197$ & $\$ 189$ \\
\hline Paraclinics & & \\
\hline Colistin-meropenem & $\$ 15$ & $\$ 11$ \\
\hline CAZ-AVI & $\$ 5$ & $\$ 4$ \\
\hline
\end{tabular}

Sensitivity analyses

To assess the robustness of the study findings, univariate sensitivity analyzes were performed to examine the impact of the key variables on the incremental cost-effectiveness ratio (ICER). The data of utilities, costs, proportion of patients who developed renal failure, treatment length and failure, and the dose of colistin were the variables selected for this analysis. Furthermore, mortality observed in the Paul study in patients with Enterobacteriaceae treated in Colistin plus meropenem was evaluated.

The probabilistic sensitivity analysis (PSA) was carried out assuming distributions for the model parameters. For clinical parameters and utilities, it was assumed that they followed Beta or Uniform distributions and, in terms of cost parameters, Gamma distribution. The results of this analysis are presented in the acceptability curve.

\section{Results}

\section{Base-Case scenario}

For the treatment of CRE infections at a follow-up time of 30 days, CAZ-AVI $(\$ 8,284)$ incurred an incremental cost per patient of $\$ 3,317$ over $\operatorname{COL}+\operatorname{MEM}(\$ 4,966)$. The general difference is mainly due to the cost associated with the treatment. However, the main cost driver for both groups was hospitalization, representing $59.92 \%$ for CAZ-AVI and $82.53 \%$ for COL + MEM of the total cost, where the percentage of people discharged is higher in the case of CAZ-AVI.

Despite the higher expected cost, CAZ-AVI showed to reduce mortality and, therefore, obtain better results in effectiveness compared to COL + MEM. In a hypothetical cohort of 1,000 patients, 92 deaths are estimated in 
the case of CAZ-AVI and 320 in the case of COL + MEM. Hence, it is estimated that CAZ-AVI generates an additional 1.76 QALY compared to COL + MEM. The model suggests that CAZ-AVI is associated with an ICER of USD 2,584/QALY.

Table 3. Events per 1,000 patients at the 30 days of follow-up

Table 3

Events per 1,000 patients at the 30 days of follow-up

\begin{tabular}{|lll|}
\hline & CAZ-AVI & Colistin-meropenem \\
\hline Death & 92 & 320 \\
\hline Alive in hospital & 724 & 607 \\
\hline Discharge home & 184 & 73 \\
\hline Additional antibiotic & 630 & 940 \\
\hline Renal failure & 50 & 130 \\
\hline
\end{tabular}

Univariate sensibility analyses

This analysis indicated that the ICER was not sensitive to changes of the variables. The variables treatment length, the utility of patients with the clinical cure, and life expectancy are those that generated the greatest variability in the results. When all model parameters were changed, the ICER was in the range of USD 1,817$3,658 /$ QALY, demonstrating that CAZ-AVI is a cost-effective strategy in all the scenarios of this analysis.

Probabilistic sensitivity analysis

This analysis showed that CAZ-AVI is more likely to be a cost-effective strategy when the threshold is higher than USD 2,438 per QALY. When these two alternatives are compared at a threshold of one Gross Domestic Product (GDP) per capita (USD 6,030) per QALY, CAZ-AVI would have a 99\% chance of being cost-effective.

Figure 2. Acceptability curve

\section{Discussion}

This is the first economic study conducted in the country related to the treatment of CRE infections with CAZAVI. Both in the baseline scenario and the sensitivity analysis, CAZ-AVI was shown as a cost-effective treatment below the thresholds usually used in Colombia, from one to three times the GDP per capita

The model reflects that management with CAZ-AVI provides a better opportunity for clinical success by decreasing the percentage of people who require additional antibiotics, reducing the number of deaths, and increasing the number of QALY compared to COL + MEM. Despite the clinical benefits of CAZ-AVI treatment, no savings are generated in the management of patients, both in treatment and hospital management. The first is due to the difference in treatment prices and the second, due to the number of people who live during 
the hospital stay. Since the mortality rate is higher in the case of COL + MEM, the use of resources in these patients is lower; in the base case, the absolute difference in the mortality rate was $23 \%$.

Given the difference in mortality among comparators, it could infer that the COL + MEM strategy will require additional financial resources at short term compared to CAZ-AVI, considering the findings of an observational study, which showed that the costs of deceased people tend to be higher than the costs of the survivors (30).

Relative to difference in terms of effectiveness and safety between colistin methane sulfonate and polymyxin $B$ there is a discussion, mainly due to the pharmacokinetic process to obtain colistin from methane sulfonate (31). From this uncertainty, a systematic review which compared both treatments and found that there is no statistical difference in mortality at 30 days or in-hospital (32). Conversely, colistin was associated to present more cases of nephrotoxicity (32). Considering the available evidence and the sensibility analysis in which the results in the ICER were not sensitive to frequency of renal failure, expect to find similar results with polymyxin $B$.

Future work will require information on the impact of methadone treatment on the cost of health care and public programs, the indirect costs incurred by patients, and adjustments to re ${ }^{-}$ect quality of life Future work will require information on the impact of methadone treatment on the cost of health care and public programs, the indirect costs incurred by patients, and adjustments to re ${ }^{-}$ect quality of life Future work will require information on the impact of randomized controlled trials evaluating antimicrobial treatment options for CRE infections and incorporated the long-term costs incurred by society, captures deathrelated costs and the inclusion of other social service costs, as productivity, emotional and behavioral disorders related with the loss of a person.

The model attempted to reflect the relevant outcomes in this type of infection for clinicians and payers, following the clinical practice of the country using the most frequent treatment and dosage for CRE as the COL-MEM. Additionally, the study adheres to the recommendations to conduct economic analysis based on the guidelines established by the Health Technology Assessment Institute in Colombia (i.e. IETS).

The clinical variables used in the model are consistent with other studies. Shields et al. reported better results of CAZ-AVI in terms of clinical success ( $85 \%$ vs $40 \%$ ) and 30 -day mortality ( $8 \%$ vs $30 \%$ ) compared to colistin (10). Likewise, Tumbarello et al. reported lower mortality with CAZ-AVI compared to other regimens as salvage therapy for CRE infections (36.5\% vs $55.8 \%$ ) (33).

Several limitations must be considered when interpreting the results in this study. The results of this analysis will be applied only to patients with similar characteristics to those of the population included in this study. Additionally, the model is based on data from an observational study (which by nature have their own limitations), due to the lack of randomized trials that reflect the efficacy of the comparative treatments. 
Another limitation is related to the long-term quality of life and survival that were extrapolated from the literature, whose extrapolation goes beyond the 30-day time horizon, which is the study follow-up from which efficacy and safety information was obtained. However, this was considered acceptable by clinical experts given the limited information in the Colombian population, in addition to being widely used in other economic studies with the same information limitation.

Having made these caveats, it is highlighted that the results found in this study are not far from what was found in another economic evaluation for this type of patient, where CAZ-AVI is found as a cost-effective strategy taking into account the thresholds established in the United States (34).

The increase in antimicrobial resistance is a reality $(35,36)$, and given the limited treatment options, there is a need for new antibiotics in the country. In this case, CAZ-AVI is presented as a therapeutic option for hospitalized patients with CRE infections, which leads to positive clinical results and decreased antibiotic use, showing itself as a cost-effective alternative compared to conventional therapy (COL $+\mathrm{MEM})$.

\section{Conclusion}

The cost-effectiveness model suggests that CAZ-AVI demonstrates an increase in QALYs and reduction in the cases of deaths, renal failure, and treatment failure, resulting in a cost-effective treatment for CRE infections compared to colistin-meropenem.

\section{Abbreviations}

\begin{tabular}{|ll|}
\hline CAZ-AVI & Ceftazidime/avibactam \\
\hline COL + MEM & Colistin in combination with meropenem \\
\hline CPI & Consumer Price Index \\
\hline CRE & Carbapenems-resistant Enterobacteriaceae \\
\hline DANE & National Administrative Department of Statistics \\
\hline GDP & Gross Domestic Product \\
\hline ICER & Incremental cost-effectiveness ratio \\
\hline ICU & Intensive Care Unit \\
\hline PSA & Probabilistic sensitivity analysis \\
\hline QALY & Quality-adjusted life years \\
\hline SECOP & Electronic System for Public Procurement \\
\hline SOAT & Mandatory Traffic Accident Service \\
\hline
\end{tabular}

\section{Declarations}


Ethics approval and consent to participate: Not applicable

Consent for publication: Not applicable

Availability of data and materials: All data generated or analyzed during this study are included in this published article

Competing interests: EL, NC and JR are paid employees of Pfizer

Funding: This work was funded by Pfizer S.A.S

Authors' contributions: NC and JR initiated and designed the study, acquired, analyzed and interpreted the data, and drafted the manuscript. EL and FV contributed to the conception and design of the study, analyzed and interpreted the data, writing and revised of manuscript. All authors read and approved the final manuscript.

Acknowledgements: This study was possible through the financial support from Pfizer S.A.S. We thank Wajeeha Ansari and Nathalie Baillon-Plot for helpful comments and excellent research assistance.

\section{References}

1. National Center for Emerging and Zoonotic Infectious Diseases/ Centers for Disease Control and Prevention (CDC). Facility Guidance for Control of Carbapenem-resistant Enterobacteriaceae (CRE). 2015.

2. Morrill HJ, Pogue JM, Kaye KS, LaPlante KL. Treatment Options for Carbapenem-Resistant Enterobacteriaceae Infections. Open forum Infect Dis [Internet]. 2015 Apr [cited 2018 Jul 17];2(2): 050. Available from: http://www.ncbi.nlm.nih.gov/pubmed/26125030.

3. Kanj SS, Kanafani ZA. Current Concepts in Antimicrobial Therapy Against Resistant Gram-Negative Organisms: Extended-Spectrum $\beta$-Lactamase-Producing Enterobacteriaceae, Carbapenem-Resistant Enterobacteriaceae, and Multidrug-Resistant Pseudomonas aeruginosa. Mayo Clin Proc [Internet]. 2011 Mar [cited 2018 Jul 17];86(3):250-9. Available from: http://www.ncbi.nlm.nih.gov/pubmed/21364117.

4. Raman G, Avendano E, Berger S, Menon V. Appropriate initial antibiotic therapy in hospitalized patients with gram-negative infections: systematic review and meta-analysis. BMC Infect Dis [Internet]. 2015 Dec 30 [cited 2018 Jul 18];15(1):395. Available from: http://www.ncbi.nlm.nih.gov/pubmed/26423743.

5. Kongnakorn T, Eckmann C, Bassetti M, Tichy E, Di Virgilio R, Baillon-Plot N, et al. Cost-effectiveness analysis comparing ceftazidime/avibactam (CAZ-AVI) as empirical treatment comparing to ceftolozane/tazobactam and to meropenem for complicated intra-abdominal infection (clAl). Antimicrob Resist Infect Control. 2019 Dec 21;8(1):1-15.

6. Carmeli Y, Armstrong J, Laud PJ, Newell P, Stone G, Wardman A, et al. Ceftazidime-avibactam or best available therapy in patients with ceftazidime-resistant Enterobacteriaceae and Pseudomonas aeruginosa complicated urinary tract infections or complicated intra-abdominal infections (REPRISE): a randomised, pathogen-directed. Lancet Infect Dis. 2016 Jun;16(6):661-73.

7. Wagenlehner FM, Sobel JD, Newell P, Armstrong J, Huang X, Stone GG, et al. Ceftazidime-avibactam Versus Doripenem for the Treatment of Complicated Urinary Tract Infections, Including Acute 
Pyelonephritis: RECAPTURE, a Phase 3 Randomized Trial Program. Clin Infect Dis. 2016 Sep;63(6):75462.

8. Mazuski JE, Gasink LB, Armstrong J, Broadhurst H, Stone GG, Rank D, et al. Efficacy and Safety of Ceftazidime-Avibactam Plus Metronidazole Versus Meropenem in the Treatment of Complicated Intraabdominal Infection: Results From a Randomized, Controlled, Double-Blind, Phase 3 Program. Clin Infect Dis. 2016;62(11):1380-9.

9. Torres A, Zhong N, Pachl J, Timsit J-F, Kollef M, Chen Z, et al. Ceftazidime-avibactam versus meropenem in nosocomial pneumonia, including ventilator-associated pneumonia (REPROVE): a randomised, doubleblind, phase 3 non-inferiority trial. Lancet Infect Dis. 2018 Mar;18(3):285-95.

10. Shields RK, Potoski BA, Haidar G, Hao B, Doi Y, Chen L, et al. Clinical Outcomes, Drug Toxicity, and Emergence of Ceftazidime-Avibactam Resistance Among Patients Treated for Carbapenem-Resistant Enterobacteriaceae Infections. Clin Infect Dis. 2016 Dec;63(12):1615-8.

11. van Duin D, Lok JJ, Earley M, Cober E, Richter SS, Perez F, et al. Colistin Versus Ceftazidime-Avibactam in the Treatment of Infections Due to Carbapenem-Resistant Enterobacteriaceae. Clin Infect Dis [Internet]. 2018 Jan 6 [cited 2018 Jul 19];66(2):163-71. Available from:

https://academic.oup.com/cid/article/66/2/163/4103289.

12. Yao X, Doi Y, Zeng L, Lv L, Liu J-H. Carbapenem-resistant and colistin-resistant Escherichia coli coproducing NDM-9 and MCR-1. Lancet Infect Dis. 2016 Mar;16(3):288-9.

13. Liu Y-Y, Wang Y, Walsh TR, Yi L-X, Zhang R, Spencer J, et al. Emergence of plasmid-mediated colistin resistance mechanism MCR-1 in animals and human beings in China: a microbiological and molecular biological study. Lancet Infect Dis. 2016 Feb;16(2):161-8.

14. Rojas LJ, Salim M, Cober E, Richter SS, Perez F, Salata RA, et al. Colistin Resistance in CarbapenemResistant Klebsiella pneumoniae: Laboratory Detection and Impact on Mortality. Clin Infect Dis. 2017;64(6):711-8.

15. Temkin E, Torre-Cisneros J, Beovic B, Benito N, Giannella M, Gilarranz R, et al. Ceftazidime-Avibactam as Salvage Therapy for Infections Caused by Carbapenem-Resistant Organisms. 2016 [cited 2020 May 5]; Available from: https://doi.org/.

16. Dimopoulos G, De Pascale G, Afonso A, Poulakou G, Karaiskos I, Lagou S, et al. The "Old"' and the 'New"' Antibiotics for MDR Gram-Negative Pathogens: For Whom, When, and How."' Front Public Heal | www.frontiersin.org [Internet]. 2019 [cited 2020 May 5];1:151. Available from: www.frontiersin.org.

17. Paul M, Daikos GL, Durante-Mangoni E, Yahav D, Carmeli Y, Benattar YD, et al. Colistin alone versus colistin plus meropenem for treatment of severe infections caused by carbapenem-resistant Gramnegative bacteria: an open-label, randomised controlled trial. Lancet Infect Dis [Internet]. 2018 Apr [cited 2019 May 31];18(4):391-400. Available from:

https://linkinghub.elsevier.com/retrieve/pii/S1473309918300999.

18. 10.1007/s00134-017-4962-2

Bassetti M, Montero JG, Paiva JA. When antibiotic treatment fails. Intensive Care Med [Internet]. 2018 Jan 14 [cited 2019 May 31];44(1):73-5. Available from: http://link.springer.com/10.1007/s00134-0174962-2. 
19. DANE. Proyecciones de la población 2005-2020. 2007 [cited 2018 Apr 16];225. Available from: https://www.dane.gov.co/files/investigaciones/poblacion/proyepobla06_20/8Tablasvida1985_2020.pdf.

20. Garcia-Hernandez A, Mayrhofer T. Quality-of-life-adjusted hazard of death: a formulation of the qualityadjusted life-years model of use in benefit-risk assessment. Value Health [Internet]. 2014 Mar 1 [cited 2018 Feb 20];17(2):275-9. Available from: http://www.ncbi.nlm.nih.gov/pubmed/24636387.

21. Song Y, Tai JHY, Bartsch SM, Zimmerman RK, Muder RR, Lee BY. The potential economic value of a Staphylococcus aureus vaccine among hemodialysis patients. Vaccine [Internet]. 2012 May 21 [cited 2019 May 31];30(24):3675-82. Available from: https://www.sciencedirect.com/science/article/pii/S0264410X12004070?via\%3Dihub.

22. $10.1086 / 318492$

Delate T, Coons SJ. The Use of 2 Health-Related Quality-of-Life Measures in a Sample of Persons Infected with Human Immunodeficiency Virus. Clin Infect Dis [Internet]. 2001 Feb 1 [cited 2019 Sep 13];32(3):e47-52. Available from: https://academic.oup.com/cid/article-lookup/doi/10.1086/318492.

23. Banco de la República de Colombia. Tasa representativa del mercado dólares [Internet]. 2019. Available from: http://www.banrep.gov.co/es/-estadisticas.

24. Consejo Nacional de. Seguridad Social en Salud. Manual Tarifario SOAT 2019. 2019.

25. Instituto del Seguro Social. Resolución 1896 de 2001. Manual de tarifas de la Entidad Promotora De Salud del Seguro Social "EPS-ISS.\&\#8221.

26. Tribiño G, Maldonado C, Segura O, Biomédica JD-. 2006 undefined. Costos directos y aspectos clínicos de las reacciones adversas a medicamentos en pacientes hospitalizados en el servicio de medicina interna de una. redalyc.org [Internet]. [cited 2019 May 31]; Available from: https://www.redalyc.org/html/843/84326105/.

27. Eficiente C. Colombia compra eficiente. colombiacompra.gov.co [Internet]. 2019; Available from: https://www.colombiacompra.gov.co/sites/cce_public/files/cce_infografias/aviso_papeleria.pdf.

28. DANE - Dirección de Síntesis y Cuentas Nacionales y Banco de la República EECB de la R-CF. PIB. Metodología año base 2005 | Banco de la República (banco central de Colombia) [Internet]. [cited 2018 Apr 19]. Available from: http://www.banrep.gov.co/es/pib.

29. Raitano M, Gabriele S, Granaglia E, Gray A. The impact of death-related costs on health-care expenditure: a survey [Internet]. 2006 [cited 2019 Jun 5]. Available from: http://aei.pitt.edu/9487/2/9487.pdf.

30. Nation RL, Velkov T, Li J. Colistin, Polymyxin B. Peas in a Pod, or Chalk and Cheese? Clin Infect Dis. 2014 Jul;59(1):88-94.

31. Vardakas KZ, Falagas ME. Colistin versus polymyxin B for the treatment of patients with multidrugresistant Gram-negative infections: a systematic review and meta-analysis. Int J Antimicrob Agents. 2017 Feb;49(2):233-8.

32. Tumbarello M, Trecarichi EM, Corona A, De Rosa FG, Bassetti M, Mussini C, et al. Efficacy of CeftazidimeAvibactam Salvage Therapy in Patients With Infections Caused by Klebsiella pneumoniae Carbapenemase-producing K. pneumoniae. Clin Infect Dis. 2019 Jan;68(3):355-64.

33. Sfeir M, Satlin M, Calfee DP, Simon MS. 1885. Cost-Effectiveness of Ceftazidime-Avibactam Compared With Colistin for Treatment of Carbapenem-Resistant Enterobacteriaceae Bacteremia and Pneumonia. 
Open Forum Infect Dis [Internet]. 2018 Nov 26 [cited 2019 Jun 5];5(suppl_1):S539-40. Available from: https://academic.oup.com/ofid/article/5/suppl_1/S539/5207575.

34. Office of Health Economics. The bacterial challenge: time to react. 2011 [cited 2018 Jul 17]; Available from: www.ecdc.europa.eu.

35. Sharma P, Towse A. New drugs to tackle antimicrobial resistance: analysis of EU policy options. 2010 [cited 2018 Jul 17]; Available from: https://papers.ssrn.com/sol3/papers.cfm?abstract_id=2640028.

\section{Figures}

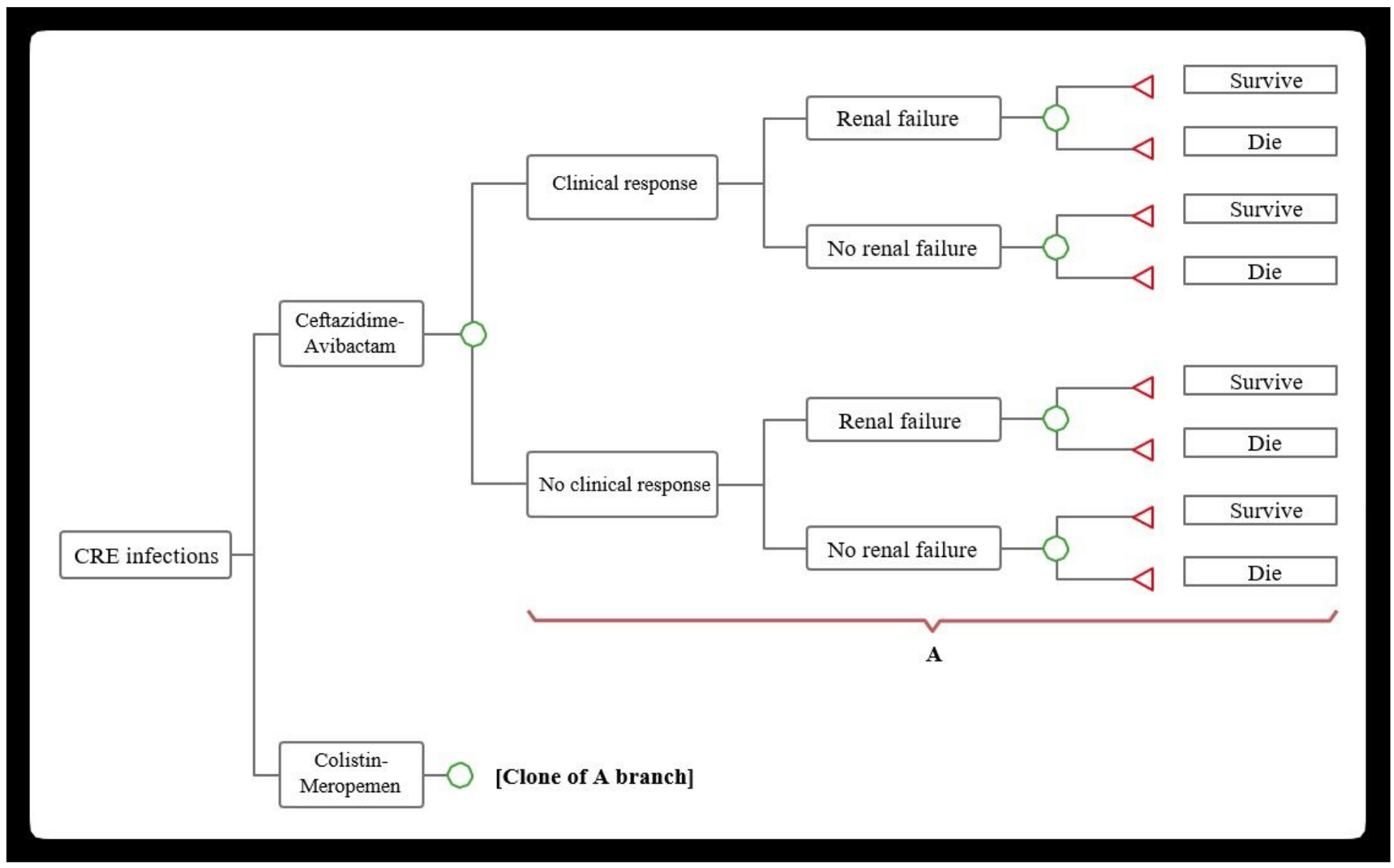

Figure 1

Model structure 


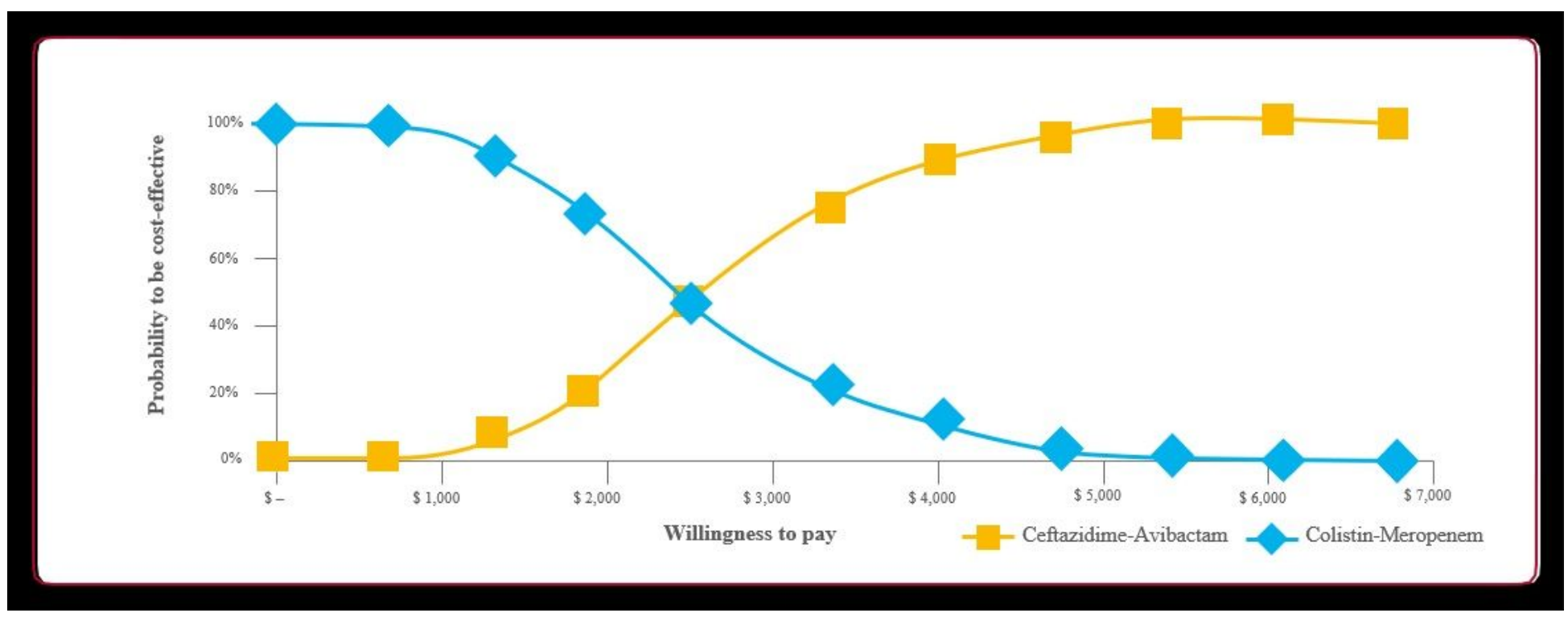

Figure 2

Acceptability curve 